\title{
Extreme Value Modelling of Rainfall Using Poisson-generalized Pareto Distribution: A Case Study Tanzania
}

\author{
Emmanuel Iyamuremye ${ }^{1}$, Joseph Mung'atu ${ }^{1}$, Peter Mwita ${ }^{2}$ \\ ${ }^{1}$ Department of Statistics and Actuarial Science, Jomo Kenyatta University of Agriculture and Technology, Nairobi, Kenya \\ ${ }^{2}$ Department of Mathematics and Statistics, Machakos University, Machakos, Kenya
}

Email address:

eiyamuremye@aims.ac.tz (E. Iyamuremye),j.mungatu@fsc.jkuat.ac.ke (J. Mung'atu), peter_mwita@yahoo.com (P. Mwita)

\section{To cite this article:}

Emmanuel Iyamuremye, Joseph Mung'atu, Peter Mwita. Extreme Value Modelling of Rainfall Using Poisson-generalized Pareto Distribution: A Case Study Tanzania. International Journal of Statistical Distributions and Applications. Vol. 5, No. 3, 2019, pp. 67-75. doi: 10.11648/j.ijsd.20190503.14

Received: July 31, 2019; Accepted: August 21, 2019; Published: September 10, 2019

\begin{abstract}
Extreme rainfall events have caused significant damage to agriculture, ecology and infrastructure, disruption of human activities, injury and loss of life. They have also significant social, economical and environmental consequences because they considerably damage urban as well as rural areas. Early detection of extreme maximum rainfall helps to implement strategies and measures, before they occur. Extreme value theory has been used widely in modelling extreme rainfall and in various disciplines, such as financial markets, insurance industry, failure cases. Climatic extremes have been analysed by using either generalized extreme value (GEV) or generalized Pareto (GP) distributions which provides evidence of the importance of modelling extreme rainfall from different regions of the world. In this paper, we focus on Peak Over Thresholds approach where the Poisson-generalized Pareto distribution is considered as the proper distribution for the study of the exceedances. This research considers also use of the generalized Pareto (GP) distribution with a Poisson model for arrivals to describe peaks over a threshold. The research used statistical techniques to fit models that used to predict extreme rainfall in Tanzania. The results indicate that the proposed Poisson-GP distribution provide a better fit to maximum monthly rainfall data. Further, the Poisson-GP models are able to estimate various return levels. Research found also a slowly increase in return levels for maximum monthly rainfall for higher return periods and further the intervals are increasingly wider as the return period is increasing.
\end{abstract}

Keywords: Extreme Value Theory, Generalized Pareto Distribution (GPD),

Poisson Generalized Pareto Distribution (Poisson-GPD), Maximum Likelihood Estimation,

Likelihood Ration Test, Exceedances

\section{Introduction}

Extreme rainfall events have caused significant damage to agriculture, ecology and infrastructure, disruption of human activities, injury and loss of life. Since such extremes significantly affect societies [1], it becomes necessary to understand and analyse changes in them. However, analysing patterns of change in extreme rainfall over Tanzania is challenging because of the variability associated with Tanzanian rainfall [2]. In addition, extreme events are rare by their definition, making it difficult to analyse or model them [3].
In recent years, floods have become more frequent in Tanzania which necessitate investigating their cause. For example, many areas are affected by heavy rainfall and the associated floods and landslides. Some examples of the loss caused by floods are the damage both to life and property experienced throughout the country during the 1997-1998 El Nino associated with floods, and the 2011 floods that wrecked the coastal city of Dar es Salaam [4]. In years [5], heavy rains accompanied with strong winds have left thousands of people displaced and without food in Muleba, Kilosa, Same and Dar es Salaam. The flooding of 2009-2010 in Kilosa proved as serious, that over three quarters of the 
farmers reported their households were affected [6]. Furthermore, in 2010, floods occurred in Kilosa (Morogoro), Mpwapwa and Kondoa (Dodoma) where more than 50000 people were affected, 5100 hectares of crops were destroyed and agricultural land was covered with mud and sand; public facilities were also destroyed [7]. According to the study [8], some towns in Tanzania, for example Dar es Salaam and Mwanza, experience floods almost every rainy season and causes significant damage. Over the last 50 years, extreme value theory has been used widely in modelling extreme rainfall and in various disciplines, such as financial markets, insurance industry, failure cases, and so on [9]. In An introduction to statistical modelling of extreme values by the paper [10], statistical models for extreme values are presented. Block maxima, for which annual maxima rainfall could be an example, converges to Generalized Extreme Value (GEV) distribution. Exceedances over threshold can be modelled by Generalized Pareto (GP) distribution, and dependence of exceedances can be modelled by de-clustering method. Return levels are derived, based on the estimation results of GEV and GP models. For non-stationary sequences, generalized linear models (GLM) are used for analysing trends in GEV and GP models [11].

Climatic extreme events have had negative impacts to both the society and economy of a country. Therefore, the early warning system require adequate understanding of climatic extreme events. So far many publications have analysed climatic extremes using either generalized extreme value
(GEV) or generalized pareto (GP) distributions which provides evidence of the importance of modelling extreme rainfall from different regions of the world: Europe [12]; France [13]; Taiwan [14]; China [15]; Australia [16]; Mozambique [16] and Bangladesh [17]. However, few studies on this subject have been carried out in Tanzania: [18, 19]. For example, the study [18] using GEV and GP distributions to define the extreme, generalized pareto distribution was found to be the best to model extreme rainfall in Tanzania. However, this study did not determine how often extreme rainfall that causes floods occurs in a certain city and during which season, rainy or dry season and intensity of such extreme rainfall was not studied.

In Tanzania, flooding has been reported in 5 regions since mid January, 2016. At least 400 people have been displaced in Dodoma municipality after 70 houses were destroyed or damaged after heavy rains between 17 and 18 January 2016. Since then, flooding has been reported in Morogoro, Katavi, Mtwara and Dar es Salaam [7]. This research devotes special attention to Tanzania because of the obvious impacts of extreme events shown in figure below. This region is considered as one of the most vulnerable regions in East Africa due to the extreme rainfall and temperature events and high rate of mortality associated to them. In this research a threshold model (known as POT model) is used to model frequency of extreme rainfall occurrences and to quantify future return level of extreme rainfall in Tanzania.

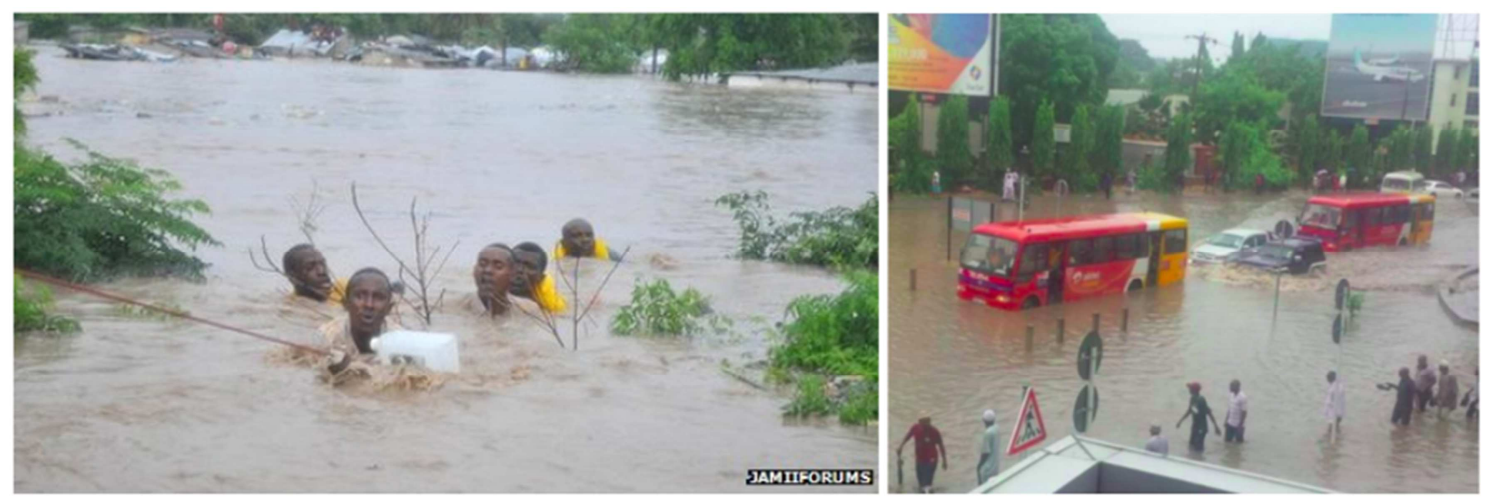

Figure 1. Rainfall in Dar-es-Salaam, Tanzania, 22 March 2015 and floods forced hundreds from their homes and roads were destroyed.

\section{Methodology}

Two methods are commonly used in modelling of extreme rainfall events; Block maxima approach and POT (Threshold exceedances) approach. The block maxima method is often wasteful of data, in particular when more data on the extremes are available, leading to large uncertainties on return level estimates. Unlike the block maxima method, the POT method provides a more efficient use of data. In the POT method, first a threshold is chosen and all the data above the threshold are being considered. Extreme rainfall is analysed by the Peaks Over Threshold (POT) approach in this study.

\subsection{Threshold Exceedances Model}

In this analysis, extremes are defined as exceedances over threshold distributed according to the generalized Pareto distribution (GPD), which is characterized by two parameters, the shape $\xi$ and the scale $\sigma \mathrm{u}$. According to [20] the POT method is a formal statistical model, consisting of a Poisson process for the occurrence of an exceedance of a high threshold and a generalized Pareto distribution for the excess over the threshold (termed "Poisson - GP model"). In this research the POT method is used because it can investigate the frequency as well as magnitude of the extremes, and is recommended for the estimation of frequency and intensity of extreme events [21]. However, one of the the limitations 
of POT is that it produces dependent data (extremes tend to occur in clusters), so we need to consider the dependence of data prior to its use. Another limitation is to make an optimal choice for the threshold $u$. A very high value of $u$ leads to few exceedances and consequently high variance estimators. On the contrary, a very low value of $\mathrm{u}$ is likely to violate the asymptotic basis of the model, leading to biases. The selection of an appropriate threshold is another critical step of the POT analysis. It is essential to choose a threshold that is high enough to be in the asymptotic limit of the distribution of exceedances, but low enough to have ample data for the fit.

\subsubsection{Generalised Pareto Distribution}

Since other values in each block are not utilised then block maxima method is somewhat wasteful and potentially important information might be lost, especially considering that extreme events are rare by definition. An alternative definition of an extreme is used, namely, that an observation is extreme if it exceeds some high threshold $\mathrm{u}$. In this case we introduce a threshold denoted by $u$. If an entire time series of daily observations are available peaks over threshold is a more suitable method than the block maxima method. Instead of splitting the data into different blocks, the peaks over threshold method is based on fitting generalized Pareto distribution to all values that exceed a certain threshold $\mathrm{u}$. The distribution for the excesses over threshold $\mathrm{u}$ is obtained by using GEV distribution as an approximation to the new distribution GPD.

\subsubsection{Poisson-GP Model for Exceedances}

The times at which the events occur can also provide important information to make the inference more exact. The Generalized Pareto (GP) distribution is the approximate distribution for excesses over a high threshold and a onedimensional Poisson process that describes the rate of exceedance. Poisson-GP model has two components.

(a) the occurrences of exceedances of some high threshold $\mathrm{u}$ (i.e., $\mathrm{X}_{\mathrm{i}}>\mathrm{u}$, for some $\mathrm{i}$ ) are generated by a Poisson process with rate parameter $\lambda$, and

(b) the excesses over threshold $u$ (i.e., $\mathrm{X}_{\mathrm{i}}-\mathrm{u}$, for some i) have a GP distribution with scale and shape parameters, $\sigma_{u}$ and $\xi$, respectively. The number of exceedances in let us say one year, follows a Poisson distribution with mean $\lambda$ and the exceedance values $Y_{1}, \ldots, Y_{n}$ are i.i.d from the GPD [21]. So, supposing $\mathrm{X}>\mathrm{u}$, the probability of the annual maximum being less than $\mathrm{x}$ for the GPD-Poisson model is

$$
P\left(x-u ; \lambda, \sigma_{u}, \xi\right)=\exp \left\{-\lambda\left[1+\xi\left(\frac{x-u}{\sigma_{u}}\right)\right]^{\frac{-1}{\xi}}\right\}
$$

\subsection{Threshold Selection}

Threshold choice involves a trade-off between bias and variance: if the threshold is too low then limiting forms may be poor models; if the threshold is too high. then there will be few observations with which to make inferences. Often, if a limiting form holds at one threshold then it holds also at all higher thresholds. Therefore, one strategy is to try several thresholds before selecting $\mathrm{u}$ to be the lowest in the range of thresholds for which estimates appear stable and models fit the data reasonably well. Procedures of threshold selection are suggested by the researchers [23, 24]. This paper considers two points to select a threshold. The first method is the use of mean residual plot, recommended by [25]. In this plot, estimator of the shape parameter should appear approximately linear in threshold $\mathrm{u}$ above a reasonable $\mathrm{u}$. Secondly, stability checking of shape parameters method is used where after choosing some values as the threshold candidates, we can then fit parameters in a GP distribution for each of the threshold. A suitable threshold can be chosen when the estimators of the shape parameter $\xi$ keep stable above the threshold.

\subsection{Parameters Estimation Using MLE}

All the models described so far will be fitted by the method of Maximum Likelihood Estimation. In this section we give a very brief overview of the main principles behind this approach, with a view towards the GP and Poisson-GP models.

\subsubsection{Inference on Generalised Pareto Model}

Setting $\mathrm{y} 1, \ldots, \mathrm{y}_{\mathrm{k}}$ to be only those values of $\mathrm{x} 1, \ldots, \mathrm{Xn}$ that exceed the threshold, the GP log-likelihood is given by

$$
\begin{gathered}
l\left(\sigma_{u}, \xi ; y_{1}, \ldots, y_{k}\right)=-k \log \sigma_{u}-\left(1+\frac{1}{\xi}\right) \sum_{i=1}^{k} \log \{1+ \\
\left.\xi\left(\frac{y_{i}}{\sigma_{u}}\right)\right\}
\end{gathered}
$$

We maximize the log likelihood function of generalized Pareto distribution to find the estimates of the parameters.

\subsubsection{Inference on Poisson-Generalised Pareto Model}

For Poisson-GP model, the likelihood function must be written in two parts, one corresponding to the poisson component and the other to the GPD. If we observed $\mathrm{N}$ exceedances $\mathrm{Y} 1, \ldots, \mathrm{YN}_{\mathrm{N}}$ over a $\mathrm{T}$-year period, then the poisson mean of $\mathrm{N}$ is $\lambda \mathrm{T}$, and the joint density of $\mathrm{N}$ and $\mathrm{Y} 1, \ldots$, $\mathrm{YN}_{\mathrm{N}}$ is

$$
\frac{(\lambda T)^{N} e^{-\lambda T}}{N !} \Pi_{i=1}^{N} h\left(Y_{i} ; \sigma_{u}, \xi\right)
$$

The log-likelihood function of the Poisson-GP model is then

$$
\begin{gathered}
l_{N, Y}\left(\lambda, \sigma_{u}, \xi\right)=N \log \lambda-\lambda T-N \log \sigma_{u}-(1+ \\
\left.\frac{1}{\xi}\right) \sum \log \left(1+\xi \frac{y_{i}}{\sigma_{u}}\right)
\end{gathered}
$$

\subsection{Estimation of Return Levels}

Assume that a generalized Pareto distribution with parameters $\sigma u$ and $\xi$ is a suitable model for exceedances of a threshold $u$ by a variable $X$. In general it is often more suitable to present the return level on an annual scale so that the $\mathrm{N}$-year return level is the level expected to be exceeded once every N years. Quantiles of the Poisson-GP model are functions of the parameters and the return period $\mathrm{Tp}$. 


$$
x_{p}=u+\sigma_{u} \log \left(\lambda T_{p}\right) \text { for } \xi=0
$$

and

$$
x_{p}=u+\frac{\sigma_{u}}{\xi}\left[1-\left(\lambda T_{p}\right)^{-\xi}\right] \text { for } \xi \neq 0
$$

\subsection{Graphic Model Diagnostics}

As the reason for fitting a statistical model to a set of data is to draw conclusions on some aspects of the population of the observed data, such conclusions could be sensitive to the accuracy of the fitted model. Thus, it is necessary to check the model accuracy and goodness-of-fit by checking its agreement with the data that were actually used to estimate it (model descriptive ability) and also checking its ability to simulate future values (model predictive ability). To check whether GP-Poisson distribution will be a good fit for our data, the assessment will be done with reference to the observed data by using the following approach:

\subsubsection{Probability Plot}

Probability plot, which is a comparison of an empirical (usually percentage rank) and the fitted distribution function. In case of perfect fit, the data would line up on the diagonal of the probability plots.

\subsubsection{Quantile Plot}

Quantile plot is also a comparison of an empirical form for estimating the exceedance and the inverse of fitted distribution. Any departure from linearity indicates model failure in perfectly fitting the data.

\subsubsection{Return Level Plot}

To use this plot as a model diagnostic, the empirical estimates of the return level function are also added. For suitable models the model based curve and empirical estimates should be in agreement.

\subsubsection{Density Function Plot}

Density plot, which is a comparison of the probability density function of a fitted model with the histogram of the POT data. This is less informative diagnostic for model as a histogram varies substantially with the choice of grouping intervals, which makes its use difficult and subjective.

\subsection{Statistical Tests}

Before carrying out extreme value modelling, the data series must meet certain statistical criteria such as independence, and stationarity. According to the study [25], the elements of annual series of short-duration rainfall may, in practice, be assumed to be independent. In some cases, however, there may be significant dependence even between annual maximum values. The identification of extremes requires the use of an independency criterion. Extreme value theory assumes total statistical independency of the sampled extremes [26] thereby providing a theoretical basis for distribution fitting.

\subsubsection{Wald and Wolfowitz Test for Independence}

The Wald and Wolfowitz (W-W) Test is a test to determine if the observations are independent. It compares the following hypotheses:

H0: The observations are independent against the alternative

H1: The observations are dependent.

\subsubsection{Stationary Test}

It is essential that the stationarity assumption be fulfilled before the classical Poisson-GP model can be fitted. The Stationary Test combines the existing tests Augmented Dickey-Fullertest (adf. test), Phillips-Perron (pp. test) and Kwiatkowski-Phillips-Schmidt-Shin Test (kpss. test) for testing the stationarity of a univariate random variables. The Stationary test compares the following hypotheses:

H0: The data is stationary and the alternative

H1: The data is non stationary.

\subsubsection{Likelihood Ratio Test}

This is a statistical test used to test the statistical significance of nested models with additional parameters. The statistical significance of the parameter of the model M0 can be assessed by the likelihood ratio test through comparison with model $\mathrm{M}$

$$
\mathrm{D}=-2\left\{1(\mathrm{M})-1\left(\mathrm{M}_{0}\right)\right\}
$$

where $1(\mathrm{M})$ and $1\left(\mathrm{M}_{0}\right)$ are the maximized log likelihood function of the models $\mathrm{M}$ and $\mathrm{M} 0$, respectively. The statistic $\mathrm{D}$ is distributed according to a Chi-square distribution, with the degree of freedom parameter being the difference in number of parameters between models $\mathrm{M}$ and $\mathrm{M}_{0}$. In this work, the likelihood ratio test is conducted for two nested extreme value distribution models. Meaning, the parameters of one model must be a subset of the parameters of the second model. Suppose the base model, Mo, is nested within the model $\mathrm{M}$. Then, if $\mathrm{P}-$ value is smaller than alpha (significance level), the decision is to reject the null hypothesis in favour of the model with more parameters.

\section{Results and Discussion}

\subsection{Statistical Description of the Data}

The analysis is based on monthly rainfall data in Tanzania available in World Meteorological Organization. The data have been recorded from 1901 up 2015 by considering available monthly rainfall data. The data set contains 1380 values of monthly rainfall. The table below shows the statistical summaries of Tanzania monthly rainfall.

Table 1. Statistical summaries of the monthly rainfall in Tanzania.

\begin{tabular}{llllllll}
\hline Variable & Period & $\mathbf{1}^{\text {st }} \mathbf{Q u}$. & Mean & 3 $^{\text {rd }}$ Qu. & Min & Max & Median \\
\hline Rainfall & $1901-2015$ & 14.200 & 83.140 & 143.600 & 1.361 & 316.100 & 71.486 \\
\hline
\end{tabular}




\subsection{Graphical Description of Tanzania Monthly Rainfall}

A box plot of the monthly rainfall has been performed, showing the variability of the rainfall for each month. In the box plot, we can see that the variability of maxima is varying over the year. The rainfall rises during the wet season (from
November to December and again from January to April) and declines during the dry period (from June to September). This graph shows that Tanzania faces extreme drought from June to September.

\subsection{Threshold Selection Techniques}

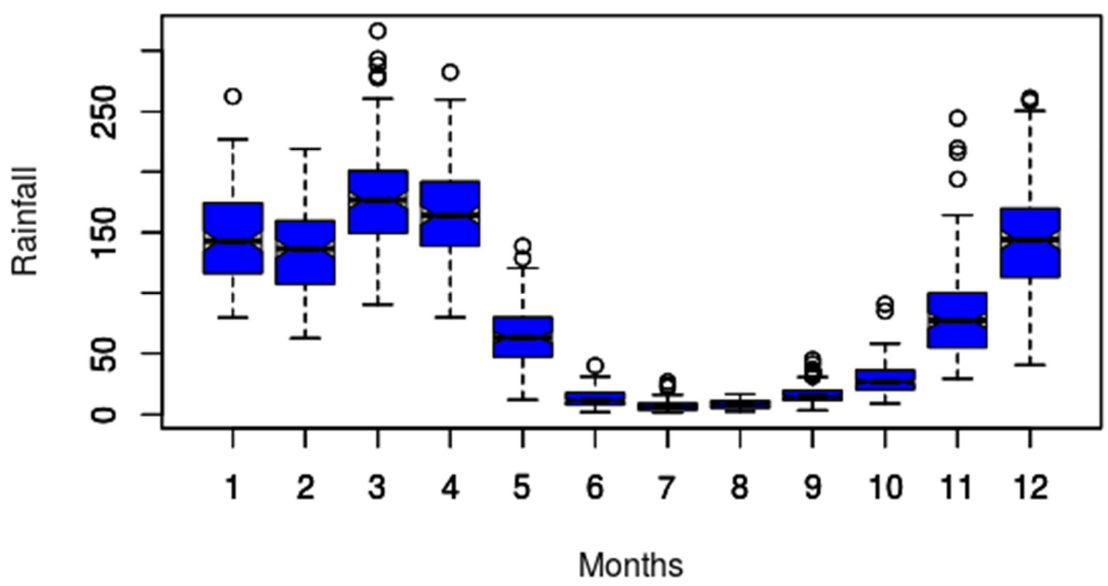

Figure 2. A boxplot of monthly rainfall.

The choice of threshold is very crucial and challenging when one wants to apply the POT method and estimate the model parameters. To analyse extreme maximum rainfall using the POT method, first a threshold value $\mathrm{u}_{0}$ is determined and then the GPD or Poisson-GPD is fitted to the rainfall values above uo. Indeed the threshold should not be too high in order to have enough data to deal with, but neither too low. Methods for selecting a threshold $\mathrm{u}_{0}$ have been used, and it is then possible to compare the different values of thresholds obtained.

\subsubsection{Mean Residual Plot}

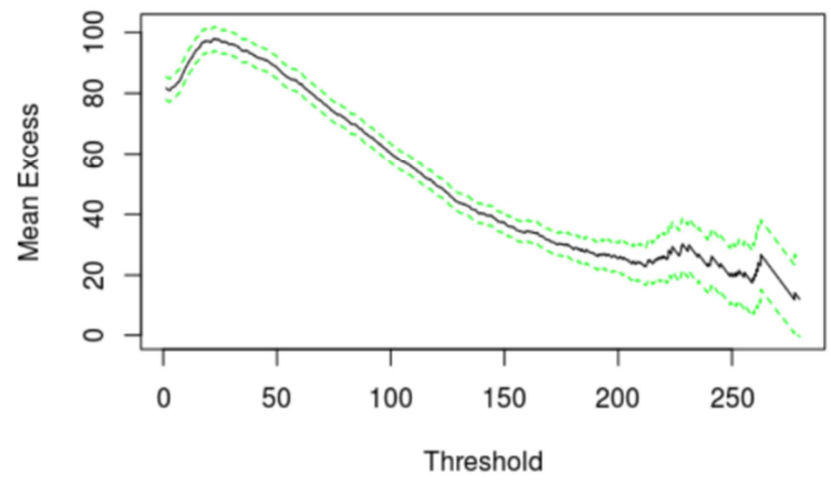

Figure 3. A Mean Residual Life Plot.

Interpretation of a mean residual life plot is not always simple in practice. The idea is to find the lowest threshold where the plot is nearly linear; taking into account the $95 \%$ confidence bounds. Mean residual life plots have been performed for Tanzania monthly rainfall (see Figure 3).

\subsubsection{Shape Parameter Stability Plot}

The second method of trying to find a threshold involves plotting the modified scale parameter and the shape parameter against the threshold $\mathrm{u}_{0}$ for a range of threshold which has been chosen to go from the $25 \%$ quantile to the $99 \%$ quantile.
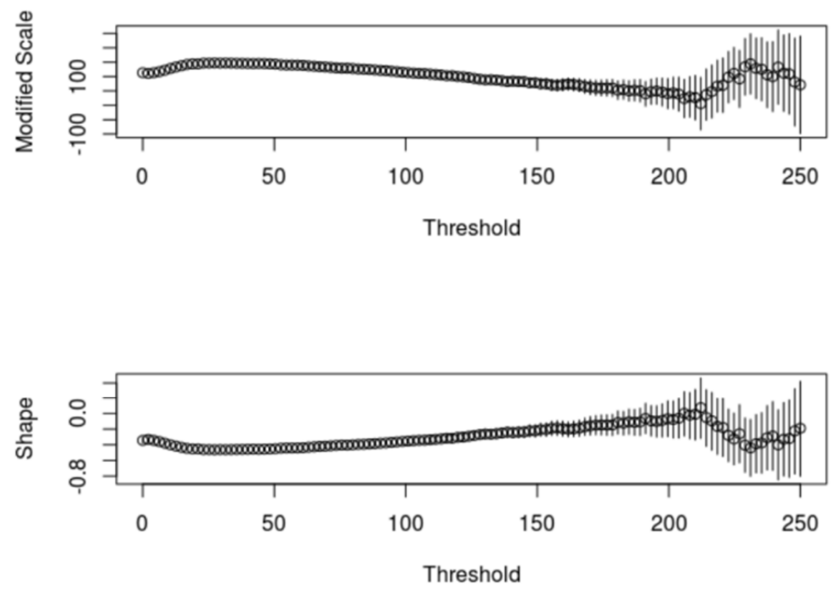

Figure 4. Shape Parameter Stability Plot.

The threshold value of $200 \mathrm{~mm}$ has been chosen using the threshold selection diagnostic plots presented above.

Two graphs showing maximum likelihood estimates and confidence intervals of the shape and modified scale parameters over a range of thresholds are produced. The parameter estimates should be stable (i.e. constant) above the threshold at which the model becomes valid. 


\subsection{Modelling Maximum Rainfall Using POT Method}

In the POT method, first a threshold is chosen and then all the data above that threshold are being considered to be extremes. Extreme values analysis was performed on this study by fitting the Poisson-generalized Pareto distribution to the sample using method of maximum likelihood estimates.

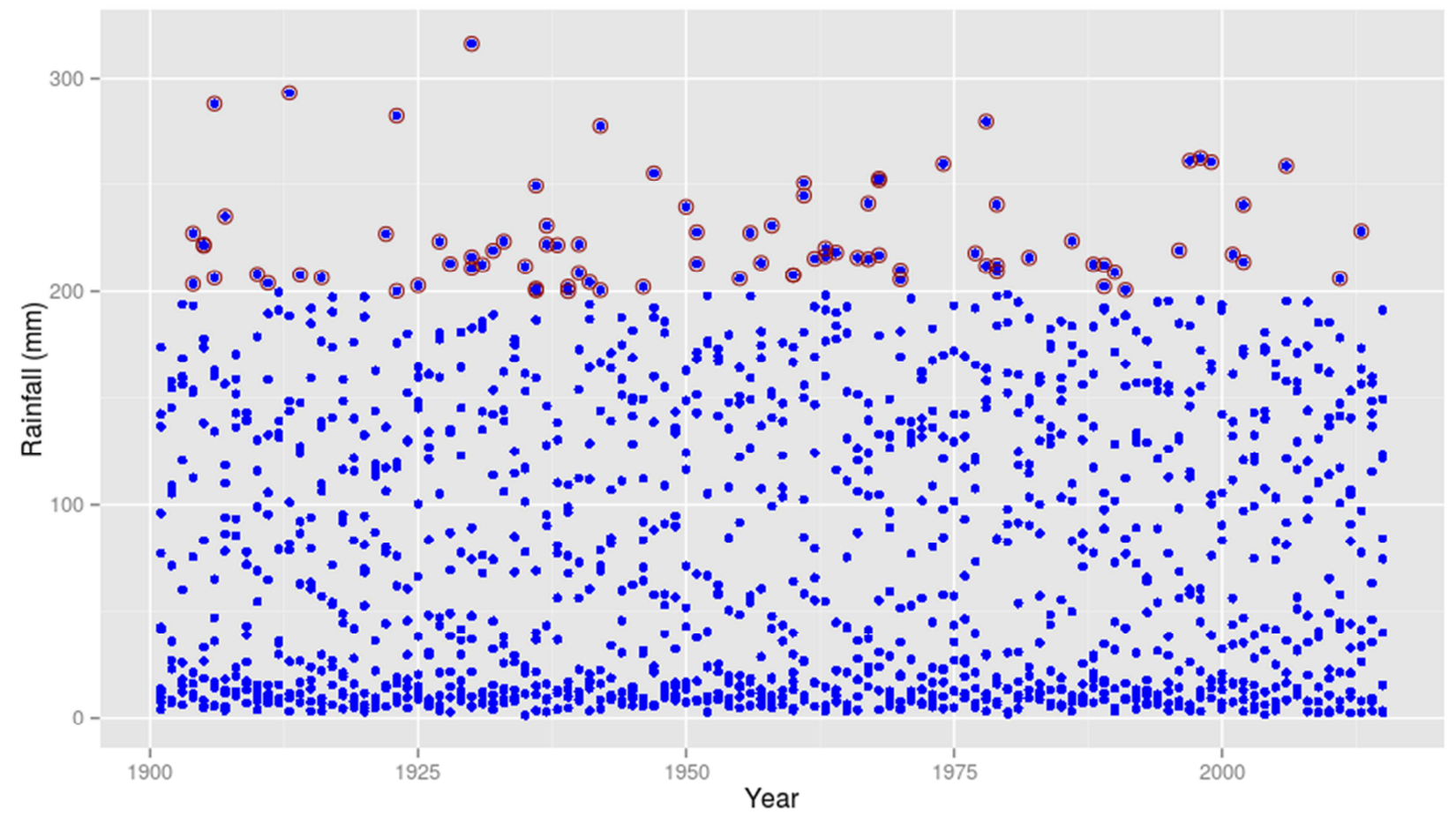

Figure 5. Monthly rainfall and excesses over threshold uo $=200 \mathrm{~mm}$.

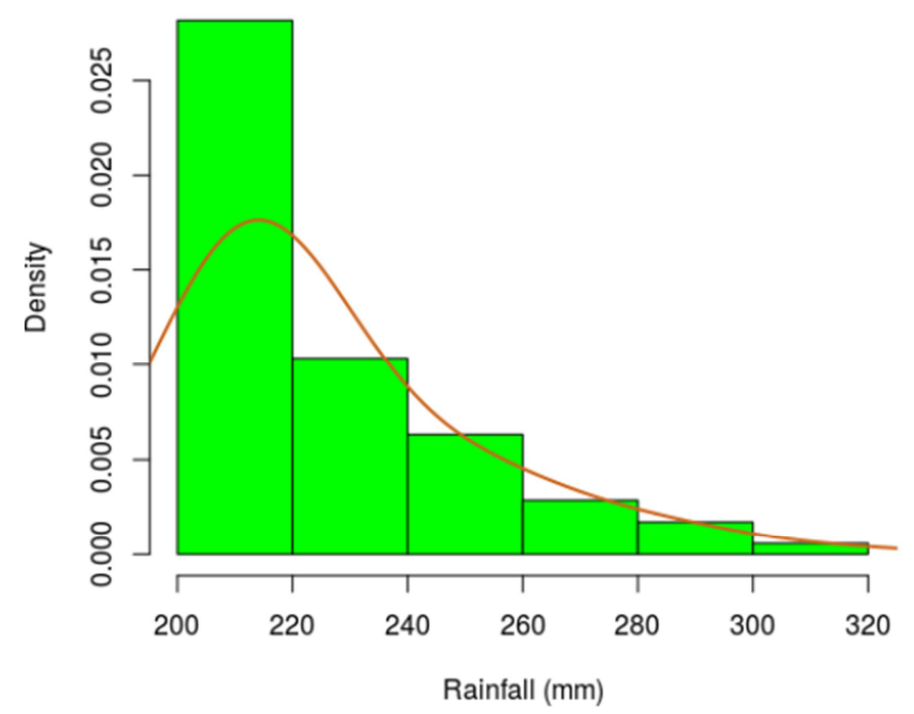

Figure 6. Histogram of rainfall exceeding threshold $u 0=200 \mathrm{~mm}$

We calculate a new data set, excess, in which the threshold is subtracted from the rainfall values. Both the original rainfall values and the calculated excesses can be seen Figure
6. The excesses over threshold are shown in red.

Some statistics for the distribution of the monthly rainfall excesses are presented below:

Table 2. Statistical summaries of Tanzania monthly rainfall excesses.

\begin{tabular}{lllllllll}
\hline Variable & Period & $\mathbf{1}^{\text {st }} \mathbf{Q u}$. & Mean & $\mathbf{3}^{\text {rd }} \mathbf{Q u}$ & Min & Max & Std & Median \\
\hline Rainfall excesses & $1901-2015$ & 208.400 & 225.400 & 232.900 & 200.100 & 316.100 & 24.354 & 217.200 \\
\hline
\end{tabular}




\subsubsection{Stationary Test}

It is important to study the stationarity of rainfall data, the sequence of random variables represents the series of maximum rainfall, it must have constant properties through time. Random variables must be independent and identically distributed satisfying stationarity assumption in order to fit Poisson-GP distribution. The aim of the test is to check if data (monthly rainfall) in time periods is stationary. Stationary test compares the null hypothesis Ho states that data are stationary while $\mathrm{H} 1$ states that they are non stationary. The KPSS test was used as a stationary test. Since the KPSS statistic value is greater than the critical value, then we do not reject the null hypothesis. Therefore, extreme monthly rainfall in Tanzania is stationary.

\subsubsection{The Poisson-generalised Pareto Distribution}

By referring to what have been discussed in the methodology, the maximum monthly rainfall data need to be fitted to a Poisson-GP distribution to forecast the occurrences of flood in Tanzania. To analyse extreme rainfall using the POT method, first a threshold value $\mathrm{u}_{0}$ is determined and then the Poisson-GPD is fitted to the rainfall values above $\mathrm{u}_{0}$. The model requires checking the stationarity and also test if Poisson-GP is a good fit for the data. A Poisson-GP with covariates model has to be employed to fit non stationary series of maximum rainfall data. Table 3 presents results of estimated parameters for the Poisson-GP model.

Table 3. Estimated parameters for Poisson-GP model.

\begin{tabular}{|c|c|c|c|c|c|c|}
\hline Threshold & Parameter & Symbol & Estimates & St. error & C. I $(95 \%)$ & $\overline{n_{u_{0}}}$ \\
\hline \multirow{3}{*}{$u_{0}=200$} & Shape & $\xi$ & -0.063 & 0.118 & $(-0.254,0.216)$ & \multirow{3}{*}{87} \\
\hline & Scale & $\sigma_{u_{0}}$ & 26.989 & 4.295 & $(19.560,36.561)$ & \\
\hline & Exceedance rate & $\lambda$ & 90.103 & 4.012 & $(79.510,98.101)$ & \\
\hline
\end{tabular}

In statistics there is a close relationship between the test of hypothesis about any parameter, say $\theta$, and the confidence interval for $\theta$. The test of size $\alpha$ of the hypothesis

$\mathrm{H}_{0}: \theta=\theta_{0}$

$\mathrm{H}_{1:} \theta=\theta_{0}$

will lead to rejection of $\mathrm{H}_{0}$ if and only if $\theta_{0}$ is not in the confidence interval for the parameter $\theta$. From Table 3 , the maximum likelihood estimate for $\xi$ is negative, what would correspond to a Poisson-Beta distribution, but the confidence interval for $\xi$ contains zero, then we fail to reject the null hypothesis, $\xi=0$.

Table 4. Estimated parameters for Poisson-exponential model.

\begin{tabular}{llllll}
\hline Threshold & Parameter & Symbol & Estimates & St. error & C. I (95\%) \\
\hline \multirow{2}{*}{$u_{0}=200$} & Scale & $\sigma$ & 25.390 & 2.722 & $(20.054,30.724)$ \\
& Exceedance rate & $\lambda$ & 86.947 & 3.002 & $(80.042,90.254)$ \\
\hline
\end{tabular}

Table 5. Deviance table analysis for Poisson-exponential and Poisson-GP model.

\begin{tabular}{llllllll}
\hline Model & M. df & Deviance & AIC & BIC & Chi-square & P-value & Significance level \\
\hline M1 & 2 & 736.510 & 740.511 & 745.443 & 0.261 & 0.609 \\
M0 & 1 & 736.070 & 738.772 & 741.234 & & 0.05 \\
\hline
\end{tabular}

Using Deviance table analysis presented above (see, Table 5), Poisson-exponential distribution is then a possible candidate to model maximum rainfall in Tanzania.

\subsubsection{Model Selection}

The value of shape parameter in Table 3 indicate that all the models obtained are classified as either Poisson-Beta distribution or Poisson-exponential distribution. The shape parameter of the distribution is negative, means that GPD distribution is Beta type, $\xi<0$. But an exponential distribution must also be considered since zero is included in the confidence interval. We select the best distribution model on basis of likelihood ration test which is described below:

Null hypothesis $\xi=0$ while alternative hypothesis $\xi=\neq 0$.

The likelihood ration test results show that Poissonexponential distribution is the best fit for our data.

\subsubsection{Graphical Model Checking}

For our data in order to check whether the model fits well the data, the assessment can be done with reference to the observed data by using the following approach: Quantile plot should show a linearity.

\subsection{Return Level Estimation}

Return levels are essentially the same as quantiles. In the case of the GPD, they are very similar, but the exceedance rate is taken into consideration. Using estimated parameters of GPD, the maximum likelihood estimator of the return level Xp. Using Poisson-exponential distribution, return level which is expected to exceed the maximum of available data is calculated for different selected return period and results are presented below: 
Probability Plot

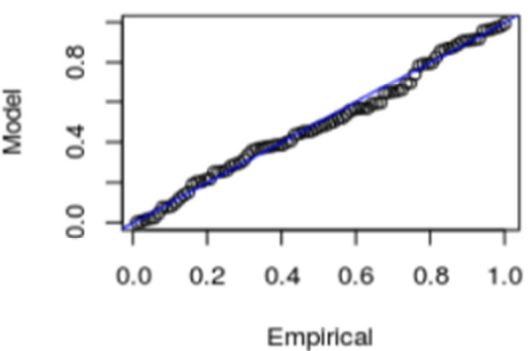

Return Level Plot

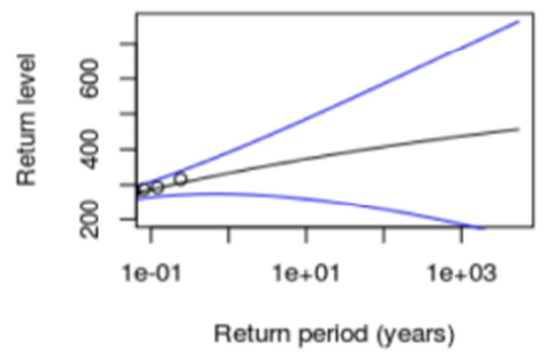

Quantile Plot

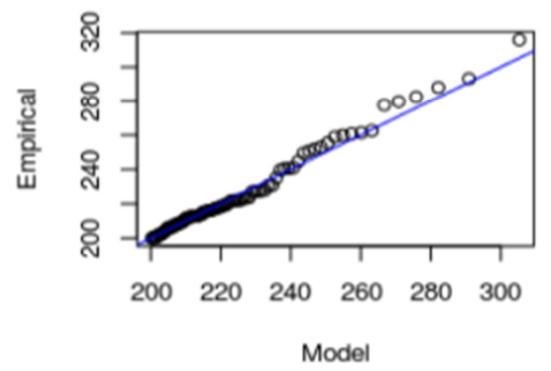

Density Plot

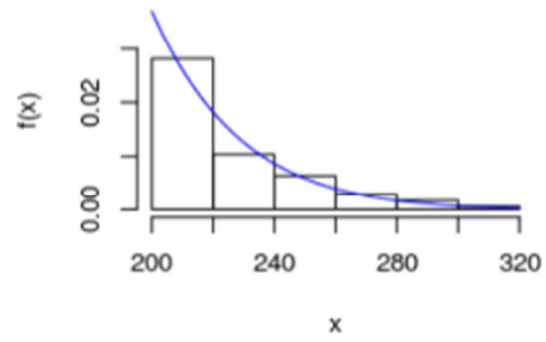

Figure 7. Model diagnostic Plots.

Departure from linearity in quantile plot also indicates model failure. A probability plot is a comparison of the empirical and fitted distribution functions should lie closer to the first diagonal. Those model checking plots are presented below.

Table 6. Return levels with corresponding return periods for Tanzania monthly rainfall.

\begin{tabular}{lllll}
\hline Variable & $\begin{array}{l}\text { Return periods } \\
\text { (Months) }\end{array}$ & $\begin{array}{l}\text { Return } \\
\text { levels }\end{array}$ & C. I (95\%) & $\begin{array}{l}\text { Significance } \\
\text { level }\end{array}$ \\
\hline & 12 & 388.541 & $(383,394)$ & \\
& 24 & 407.249 & $(402,412)$ & \\
& 36 & 418.192 & $(413,423)$ & \\
Monthly & 68 & 425.956 & $(421,431)$ & \\
Rainfall & 72 & 431.978 & $(427,437)$ & 0.05 \\
& 84 & 436,899 & $(432,442)$ & \\
& 96 & 441.059 & $(436,446)$ & \\
& 108 & 444.663 & $(440,450)$ & \\
& 120 & 447.842 & $(443,453)$ & \\
\hline
\end{tabular}

\section{Conclusions}

After selecting suitable threshold, the data is fitted to the Poisson-GP model M1 and Poisson-exponential model M0. Likelihood ratio test is then used to select the preferred model for maximum monthly rainfall data. The likelihood ratio test, revealed that $\mathrm{M} 0$ is preferred over M1. This test agreed with Deviance Analysis Table by considering BIC, AIC and $\mathrm{P}-$ value obtained. The confidence interval confirms this conclusion. Hence, the Poisson-exponential distribution was found to be more suitable for extreme monthly rainfall in Tanzania. Poisson-exponential model was used to estimate the return levels for maximum rainfall. Further, we found that the return levels for maximum monthly rainfall increase slowly for higher return periods and further the intervals are increasingly wider as the return period is increasing. A typical question is to estimate what is the amount of rain on one month that is expected to be exceeded once every $\mathrm{T}$ years. Mathematically speaking, the problem is to estimate the T-years quantile $\mathrm{q}(1 /(365 \times 12 \mathrm{~T}))$ of the monthly rainfall. For example, from table above, the amount of rain on one month that is expected to be exceeded once every 100 years is $450.686 \mathrm{~mm}$.

\section{References}

[1] Trends in summer extreme temperatures over the iberian peninsula using nonurban station data. Acero, F., Garc 1a, J. A., Gallego, M. C., Parey, S., \& Dacunha-Castelle, D. (2014). Journal of Geophysical Research: Atmospheres, 119 (1), 3953.

[2] Extreme value analysis of wet and dry periods in sicily. Bordi, I., Fraedrich, K., Petitta, M., \& Sutera, A. (2007). Theoretical and Applied Climatology, 87 (1-4), 61-71.

[3] Extreme value analysis of wet and dry periods in sicily. Bordi, I., Fraedrich, K., Petitta, M., \& Sutera, A. (2007). Theoretical and Applied Climatology, 87 (1-4), 61-71.

[4] Extreme rainfall analysis at ungauged sites in the south of france: comparison of three approaches. Carreau, J., Neppel.

[5] L., Arnaud, P., \& Cantet, P. (2013). Journal de la Societe Francaise de Statistique, 154 (2), 119-138.

[6] Statistical modelling of recent changes in extreme rainfall in taiwan. Chu, L.-F., McAleer, M., \& Wang, S.-H. (2014). International Journal of Environmental Science and Development, 4 (1), 52-55. 
[7] Modelling non-stationary annual maximum flood heights in the lower limpopo river basin of Mozambique. Maposa, D., Cochran, J. J., \& Lesaoana, M. (2016). Jamba: Journal of Disaster Risk Studies, 8 (1).

[8] Modeling non-stationarity in intensity, duration and frequency of extreme rainfall over India. Mondal, A. \& Mujumdar, P. P. (2015). Journal of Hydrology, 521, 217-231.

[9] Changes in the extreme daily rainfall in south Korea. Park, J.S., Kang, H.-S., Lee, Y. S., \& Kim, M.-K. (2011). International Journal of Climatology, 31 (15), 2290-2299.

[10] An introduction to statistical modelling of extreme values. Omey, E., Mallor, F., \& Nualart, E. (2009). Application to calculate extreme wind speeds.

[11] Extreme events in Pakistan. Zahid, M. (2017): Physical processes and impacts of changing climate.

[12] Effect of the occurrence process of the peaks over threshold on the flood estimates. nz, B. \& Bayazit, M. (2001). Journal of Hydrology, 244 (1), 86-96.

[13] Evidence of trend in return levels for daily rainfall in new Zealand. Withers, C. S. \& Nadarajah, S. (2000). Journal of Hydrology (New Zealand), 155-166.

[14] Statistics of extremes, with applications in environment, insurance, and finance. Smith, R. L. (2003). In Extreme values in finance, telecommunications, and the environment (pp. 20 97). Chapman and Hall/CRC.

[15] Non homogeneous poisson process modelling of seasonal extreme rainfall events in Tanzania. Ngailo, T., Shaban, N., Reuder, J., Rutalebwa, E., \& Mugume, I. (2016b). International Journal of Science and Research (IJSR), 5 (10), 1858-1868.

[16] Modelling of extreme maximum rainfall using extreme value theory for Tanzania. Ngailo, J., Reuder, J., Rutalebwa, E., Nyimvua, S., \& Mesquita, D. (2016a). Int. J. Sci. Innov. Math. Res, 4, 34-45.

[17] Extremes of daily rainfall in west central Florida. Nadarajah, S. (2005). Climatic change, 69 (2-3), 325-342.
[18] Long-term changes in the frequency, intensity and duration of extreme storm surge events in southern europe. Cid, A., Meńendez, M., Castanedo, S., Abascal, A. J., M endez, F. J., \& Medina, R. (2016). Climate dynamics, 46 (5-6), 1503-1516.

[19] An introduction to statistical modeling of extreme values. Coles, S., Bawa, J., Trenner, L., \& Dorazio, P. (2001)., volume 208. Springer.

[20] Statistics of extremes. Annual Review of Statistics and its Application. Davison, A. C. \& Huser, R. (2015), 2, 203-235.

[21] Statistical modeling of hot spells and heat waves. Furrer, E. M., Katz, R. W., Walter, M. D., \& Furrer, R. (2010). Climate Research, 43 (3), 191-205.

[22] Models for exceedances over high thresholds. Davison, A. C. $\&$ Smith, R. L. (1990). Journal of the Royal Statistical Society. Series B (Methodological), 52 (3), 393-442.

[23] A two-step framework for over-threshold modelling of environmental extremes. Bernardara, P., Mazas, F., Kergadallan, X., \& Hamm, L. (2014). Natural Hazards and Earth System Sciences, 14 (3), 635-647.

[24] Extreme value modeling of precipitation in case studies for china. Ender, M. \& Ma, T. (2014). International Journal of Scientific and Innovative Mathematical Research (IJSIMR), 2 (1), 23-36.

[25] The frequency distribution of the annual maximum (or minimum) values of meteorological elements. Jenkinson (1955). Quarterly Journal of the Royal Meteorological Society, 81 (348), 158-171.

[26] Statistics of extremes in hydrology. Katz, R. W., Parlange, M. B., \& Naveau, P. (2002). Advances in water resources, 25 (812), 1287-1304.

[27] Statistical modeling of extreme rainfall in southwest western Australia. Li, Y., Cai, W., \& Campbell, E. (2005). Journal of climate, 18 (6), 852-863. 